\title{
Rationally Designing Simple Rod-Like Amphiphilic NIR-Emissive AIE Probes for Precise In-Vivo Detection of A $\beta$ Fibrils/Plaques at A Super-Early Stage
}

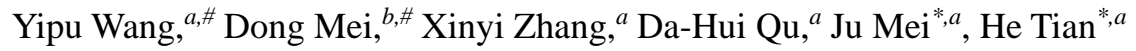 \\ ${ }^{a}$ Key Laboratory for Advanced Materials, Feringa Nobel Prize Scientist Joint Research Center, \\ Joint International Research Laboratory for Precision Chemistry and Molecular Engineering, \\ Institute of Fine Chemicals, School of Chemistry \& Molecular Engineering, East China University \\ of Science \& Technology, 130 Meilong Road, Shanghai 200237, P. R. China \\ ${ }^{b}$ Clinical Research Center, Beijing Children's Hospital, Capital Medical University, National \\ Center for Children's Health, Beijing 100045, China \\ ${ }^{\#}$ These authors contribute equally \\ *E-mail address: daisymeiju@ecust.edu.cn; tianhe@ecust.edu.cn
}

\begin{abstract}
With increase of social aging, Alzheimer's disease (AD) has been one of the serious diseases threatening human health. The occurrence of $\mathrm{A} \beta$ fibrils or plaques is recognized as the hallmark of $\mathrm{AD}$. Currently, optical imaging has stood out to be a promising technique for the imaging of $\mathrm{A} \beta$ fibrils/plaques and the diagnosis of $\mathrm{AD}$. However, restricted by their poor blood-brain barrier (BBB) penetrability, short-wavelength excitation and emission, and aggregation-caused quenching (ACQ) effect, the clinically used gold-standard optical probes such as thioflavin T (ThT) and thioflavin $\mathrm{S}$ (ThS), are not effective enough in the early diagnosis of AD in vivo. Herein, we put forward an "all-in-one" design principle and demonstrate its feasibility in developing high-performance fluorescent probes which are specific to $\mathrm{A} \beta$ fibrils/plaques and promising for super-early in-vivo diagnosis of AD. As a proof of concept, a simple rod-like amphiphilic NIR fluorescent AIEgen, i.e., AIE-CNPy-AD, is developed by taking the specificity, BBB penetration ability, deep-tissue penetration capacity, high signal-to-noise ratio (SNR) into consideration. AIE-CNPy-AD is constituted by connecting the electron-donating and accepting moieties through single bonds and tagging with a propanesulfonate tail, giving rise to the NIR fluorescence, aggregation-induced emission (AIE) effect, amphiphilicity, and rod-like structure, which in turn result in high binding-affinity and excellent specificity to $\mathrm{A} \beta$ fibrils/plaques, satisfactory ability to penetrate $\mathrm{BBB}$ and deep tissues, ultrahigh SNR and sensitivity, and high-fidelity imaging capability. In-vitro, ex-vivo, and in-vivo identifying of $\mathrm{A} \beta$ fibrils/plaques in different strains of
\end{abstract}


mice indicate that AIE-CNPy-AD holds the universality to the detection of $\mathrm{A} \beta$ fibrils/plaques. It is noteworthy that AIE-CNPy-AD is even able to trace the small and sparsely distributed $\mathrm{A} \beta$ fibrils/plaques in very young AD model mice such as 4-month-old APP/PS1 mice which are reported to be the youngest mice to have $\mathrm{A} \beta$ deposits in brains, suggesting its great potential in diagnosis and intervention of $\mathrm{AD}$ at a super-early stage.

\section{Keywords}

aggregation-induced emission (AIE), Alzheimer's disease (AD), $\beta$-Amyloid fibrils, molecular probes, in-vivo imaging, NIR fluorescence

\section{INTRODUCTION}

Alzheimer's disease (AD) is a degenerative disease of the nervous system, which will lead to memory loss, behavior disorder, cognitive decline, and eventually death. ${ }^{1-3}$ According to the statistic, there are about 50 million patients with Alzheimer's disease all over the world. It is predicted that the number of $\mathrm{AD}$ patients will increase to 150 million by 2050 , exerting serious social and economic burden to countries worldwide. ${ }^{4,5}$ Currently, clinical diagnosis of AD patients is mainly through the combination of the inquiring of the patients' genetic history, neuropsychiatric test, neuropathological diagnosis, and neuroimaging diagnosis. Only after death of $\mathrm{AD}$ patients, can final diagnosis is confirmed by brain tissue examination. ${ }^{6,7} . \beta$-Amyloid $(\mathrm{A} \beta)$ hypothesis has shown that the extracellular accumulation of $\beta$-amyloid peptides forming fibrotic plaques is one of the neuropathological hallmarks of $\mathrm{AD} \cdot{ }^{8-13} \mathrm{~A} \beta$ fibrils have strong neurotoxicity which will result in death of neuronal cells and damage of nervous system, so early detection of A $\beta$ fibrils and plaques in vivo with low damage plays extremely important role in the diagnosis and subsequent treatment and prevention of $\mathrm{AD} .^{9,14-18}$

Undoubtedly, it is of great significance to detect $\mathrm{A} \beta$ fibrils under complex biological environments and image $\mathrm{A} \beta$ plaques in vivo. ${ }^{19,20} \mathrm{By}$ far, a large number of imaging techniques have been utilized to clinically diagnose $\mathrm{AD}$, such as magnetic resonance imaging (MRI), positron emission tomography (PET), single photon emission computed tomography (SPECT). ${ }^{21}$ Nevertheless, MRI only can image larger plaques because of its limited sensitivity; PET needs radioisotopes which are hard to be obtained and probably have radioactive exposure danger to 
patients; and SPECT has relatively higher background and the tracers used in this technique usually have poor BBB penetration ability. ${ }^{1,6,15,22,23}$ Drawbacks of these techniques hamper their wide application in early diagnosis of $\mathrm{AD} .{ }^{11}$ Compared with the above techniques, fluorescence imaging technique enjoys real-time and in-situ monitoring ability, high sensitivity, low biological toxicity, non-invasiveness, superior spatiotemporal resolution, low cost, and technical simplicity, making it promising in the imaging of $\mathrm{A} \beta$ fibrils and diagnosis of $\mathrm{AD}^{1,7,11,23,24}$

ThT, ThS, and indocyanine green (ICG) are the most commonly used fluorescent dyes to histologically stain $\mathrm{A} \beta$ fibrils and plaques. ${ }^{25}$ However, the excessively excellent water-solubility of these dyes limits their ability of penetrating the blood-brain barrier (BBB), ${ }^{26,27}$ rendering them merely effective in in-vitro and ex vivo imaging of $\mathrm{A} \beta$ fibrils/plaques. Moreover, ThT is hardly able to distinguish $\mathrm{A} \beta$ peptides of different aggregation levels. ${ }^{28}$ The short-wavelength excitation and emission of probes like ThT and ThS prevent them to be used for in-vivo imaging, because of the unsatisfactory deep-tissue penetration ability and non-negligible phototoxicity to organisms. In addition, most of these fluorophores suffer from aggregation-caused quenching (ACQ) effect that results in self-quenching of FL signal after binding to $\mathrm{A} \beta$ species and the reduced detection sensitivity and imaging resolution. ${ }^{29}$ These objective factors such as the poor specificity, less satisfactory sensitivity, small Stokes shift, relatively low reliability make these probes difficult to ensure their effectiveness in in-vivo imaging. ${ }^{7,8,30}$ Sensitive and reliable probes with high ability to penetrate $\mathrm{BBB}$ and deep tissues which can be employed to detect $\mathrm{A} \beta$ fibrils and image $\mathrm{A} \beta$ plaques in vivo is urgently desirable. ${ }^{31}$

By analyzing the structure and performance of the small molecule fluorescent probes reported to detect $\mathrm{A} \beta$ fibrils and plaques, it can be found that the fluorescent probes having rod-like geometric configurations and donor-acceptor (D-A) electronic structures usually perform relatively well (Scheme 1A). ${ }^{1,2,14,26,32}$ On the one hand, the rod-like structured probes have a certain similarity with $\mathrm{A} \beta$ fibrils in morphology, which is conducive to the binding of probes and $\mathrm{A} \beta$ fibrils and subsequently benefits the specificity. On the other hand, the probes with D-A effect are sensitive to the environmental hydrophobicity, with the emissions intensified when bound to hydrophobic areas of amyloids abundant in $\beta$-sheet structure. ${ }^{33-35}$ Though these two essential structural features can guarantee the basic performance of the probes, other deficiencies still seriously affect the detection and imaging performance of these probes. Most of the reported 
probes shown ACQ effect, like ICG, resulting in high background and low SNR. Moreover, simple D-A structure in probes like ThT cannot ensure red or NIR emission which can avoid the interference by auto-fluorescence of organisms and improve the penetration ability of deep tissue.

Based on the analysis above, we sort out four main criteria which should be satisfied to realize high-performance fluorescent probes for detecting and imaging $\mathrm{A} \beta$ fibrils and plaques in vivo at an early stage (Scheme 1B): (1) geometric configurations matching the $\beta$-sheet structure; (2) balanced hydrophilicity and hydrophobicity which guarantees the BBB crossing ability and high SNR; (3) strong D-A effect ensuring fluorescent response, long-wavelength excitation and emission, and large Stokes shift; (4) anti-ACQ effect that enhances the fluorescent response, SNR, and imaging resolution. In accordance with these criteria, we propose a systematic "all-in-one" strategy for rationale design of high-performance in-vivo imaging contrast agents for precise detection of $\mathrm{A} \beta$ fibrils/plaques. In other words, rod-like geometric configurations, hydrophilic unit-decorated hydrophobic skeleton, large D- $\pi$-A electronic structure, and $3 \mathrm{D}$ flexible conformation are ingeniously integrated in one molecule to meet the above four criteria (Scheme 1B).

As a proof of concept, AIE-CNPy-AD is designed following the "all-in-one" strategy. In this molecule, electron-donating dimethylamino, electron-accepting acetonitrile and pyridyl group are linked together via single bonds and bridged by benzyl groups and $\mathrm{C}=\mathrm{C}$ double bond, affording a large 3D rod-like configured D- $\pi$-A framework. Benefited from such a framework, strong $\mathrm{A} \beta$ fibril/plaque-binding capability, efficient aggregation-induced emission (AIE) effect ${ }^{36}$ and bright red/NIR fluorescence are achieved. Furthermore, the hydrophilic propanesulfonate group is attached to the hydrophobic molecular backbone to generate the zwitterionic and amphiphilic AIE-CNPy-AD, endowing AIE-CNPy-AD with low background, high SNR and good BBB penetration ability. Since as compared with traditional ACQ probes, AIE probes often have the advantages of good photo-stability, high SNR, and resistance to photo-bleaching, the imaging performance of the fluorescent probes is greatly improved. ${ }^{37-40}$ As a result, the rod-like amphiphilic NIR-emissive AIE probe AIE-CNPy-AD possesses extremely excellent specificity to A $\beta$ fibrils/plaques, superb deep-tissue penetrating ability, good anti-interference by auto-fluorescence from organisms, improved photo-stability, superior BBB penetrating capability, and high-resolution and high-contrast imaging ability. 


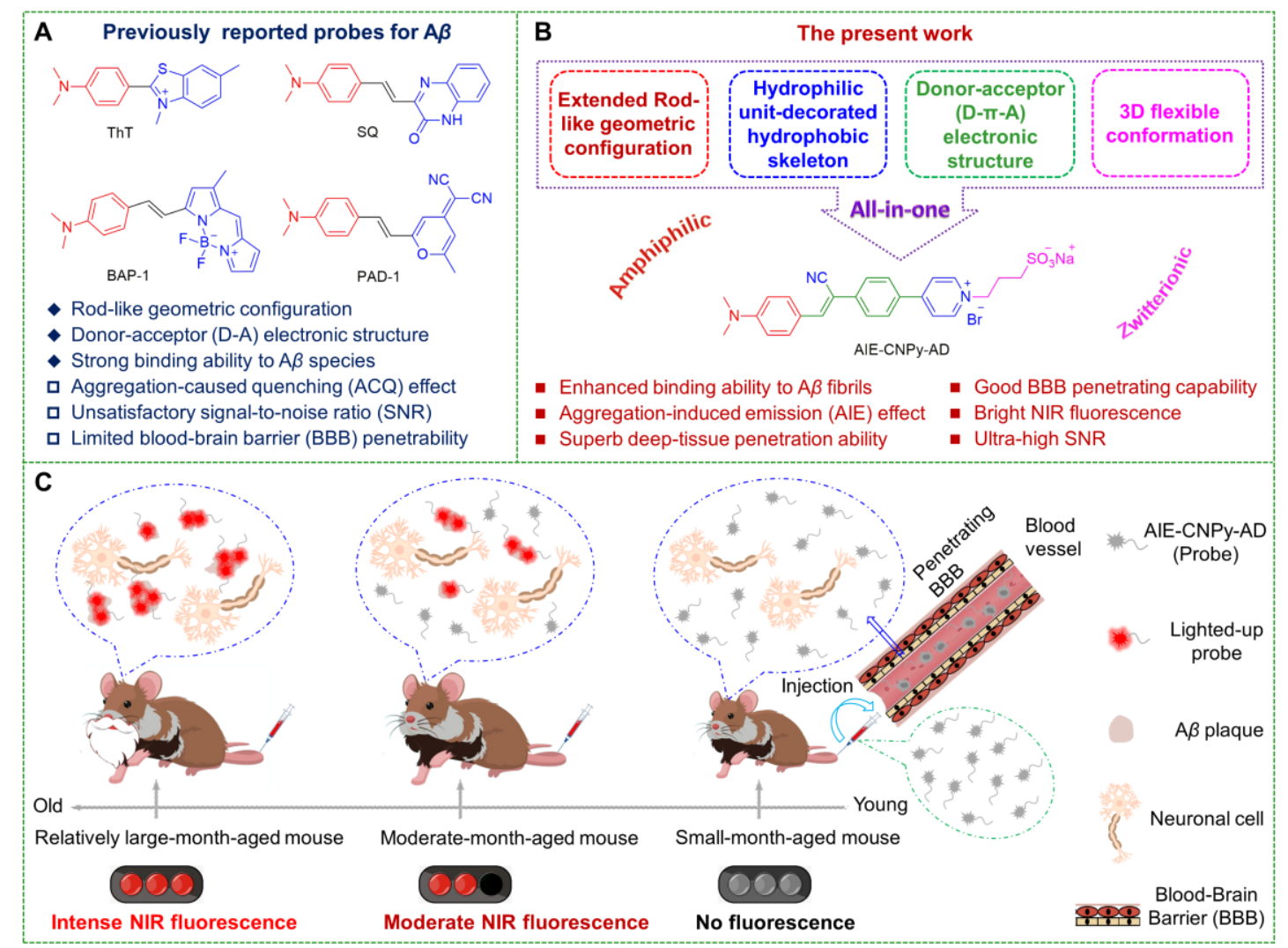

Scheme 1. (A) Representatives of typical probes for $\mathrm{A} \beta$ detection with rod-like configuration and D-A electron structure. (B) Our red/NIR AIE probe (AIE-CNPy-AD) for A $\beta$ fibrils/plaques rationally constructed based on the proposed "all-in-one" design strategy. (C) Schematic illustration of the in-vivo imaging of $\mathrm{A} \beta$ plaques with AIE-CNPy-AD in brains of AD model mice in different age periods via tail vein injection, and the potential of AIE-CNPy-AD in super-early diagnosis of AD.

With the aid of the above extraordinary characteristics, the elaborated probe AIE-CNPy-AD is able to realize detection of $\mathrm{A} \beta$ fibrils in vitro with ultra-high SNR and in-situ mapping of $\mathrm{A} \beta$ plaques in vivo with ultra-sensitivity, and high fidelity and contrast. More importantly, the precise in-situ and in-vivo mapping ability of $\mathrm{A} \beta$ plaques is not limited by mouse strains. It is worth mentioning that even small and sparsely distributed $\mathrm{A} \beta$ plaques in the brains of $\mathrm{AD}$ transgenic mice APP/PS1 as young as 4-months old could be visualized by our probe. It is reported that the $\mathrm{A} \beta$ plaques would not appear when the APP/PS1 mice are younger than 4-months old. Moreover, the increase and enlargement of $\mathrm{A} \beta$ plaques as well as the progression of $\mathrm{AD}$ as the mice grow could be clearly revealed by the present probe. In other words, AIE-CNPy-AD is very promising in super-early diagnosis and highly reliable progression monitoring of $\mathrm{AD}$ (Scheme 1C). To the best of our knowledge, this is the first work of harnessing the "all-in-one" strategy to rationally 
design AIE-active NIR imaging contrast agent for light-up and in-situ tracing of $\mathrm{A} \beta$ plaques in mice of different strains and ages. What's more, the probe maps the $\mathrm{A} \beta$ plaques at the earliest stage as compared to all the reported fluorescent probes.

\section{RESULTS AND DISCUSSION}

Rational Design and Facile Synthesis of AIE-CNPy-AD. We make full use of the "all-in-one" strategy to pursuit high-performance probes for super-early in-situ tracing of $\mathrm{A} \beta$ fibrils in vivo. As introduced above, extended D- $\pi$-A electronic architecture is built by utilizing the dimethylamino as electron-donor, the acetonitrile and pyridyl group as electron-acceptor, single bonds as linkers, and benzyl groups and $\mathrm{C}=\mathrm{C}$ double bond as $\pi$-bridges, respectively. Red or even NIR emission and relatively long-wavelength excitation is supposed to result from this D- $\pi$-A structured backbone. Such a rod-like geometric configuration is envisioned to bestow the probe with strong binding affinity to $\mathrm{A} \beta$ fibril/plaque. Moreover, according to the principle of restriction of intramolecular motions (RIM), the multiple rotors in the skeleton would efficiently consume the excited-state energy and lead to weak or even no emission in the unconstrained state, while the distorted 3D conformation would prevent $\pi-\pi$ stacking and self-quenching in the constrained state. It means such an AIE-active probe would give light-up response to the target species with satisfactory SNR under suitable conditions. To further reinforce the SNR, hydrophilic propanesulfonate group is incorporated to the hydrophobic skeleton to for one thing enhance the solubility of the probe in aqueous media and thus reduce the background/noise signal, and for another to strengthen the binding between the probe and $\mathrm{A} \beta$ fibrils/plaques via multiple electrostatic interactions. Moreover, the considerable lipophilicity of the AIE-CNPy-AD is anticipated to be sufficient to ensure desirable BBB penetrability. The elaborately designed rod-like amphiphilic NIR-emissive zwitterionic AIE probe AIE-CNPy-AD was conveniently synthesized according to the synthetic route shown in Scheme S1 in the Electronic Supporting Information (ESI) with chimerically available cheap raw materials. AIE-CNPy-AD was fully characterized with the help of ${ }^{1} \mathrm{H}$ (Figure S1), ${ }^{13} \mathrm{C}$ NMR (Figure S2) and high-resolution mass spectrometry (HRMS, Figure S3).

\section{Excellent Photophysical Properties Facilitating the Detection of A $\beta$}

Fibrils/Plaques. AIE-CNPy-AD showed an absorption maximum at $455 \mathrm{~nm}$ and an emission 
maximum at $720 \mathrm{~nm}\left(10^{-4} \mathrm{M}\right)$ in DMSO solution (Figure 1A), with the Stokes shift of as large as $265 \mathrm{~nm}$. As AIE-CNPy-AD is soluble in highly polar solvents but aggregates in less polar solvents, DMSO was chosen as a good solvent, and THF was selected as a poor solvent to evaluate the AIE behaviors of AIE-CNPy-AD (Figure 1 and Figure S4). As anticipated, with the addition of THF, fluorescence enhancement was clearly observed, with the emission peak blue-shifted from $720 \mathrm{~nm}$ $($ DMSO solution) to $675 \mathrm{~nm}(\mathrm{DMSO} / \mathrm{THF}=1 / 99, \mathrm{v} / \mathrm{v})$, manifesting the synergistic effect of AIE and intramolecular charge transfer (ICT) (Figure 1B). The emission intensity continuously increased slowly when the THF fraction $\left(f_{\mathrm{THF}}\right)$ was no more than $60 \mathrm{vol} \%$, while boosted sharply once $f_{\text {THF }}$ reached 70 vol\% (Figure 1C). Remarkably, AIE-CNPy-AD was hardly affected by the $\mathrm{pH}$ value varying from 3 to 10 covering the normal $\mathrm{pH}$ range of human body (Figure S5 and Figure 1D), suggesting the good $\mathrm{pH}$ stability of the fluorescence emission properties of our probe. Taken together, all these results undoubtedly demonstrated the remarkable AIE feature, strong ICT effect, and the stable NIR fluorescence in the aggregated state of AIE-CNPy-AD which are conducive to the imaging of $\mathrm{A} \beta$ deposits.
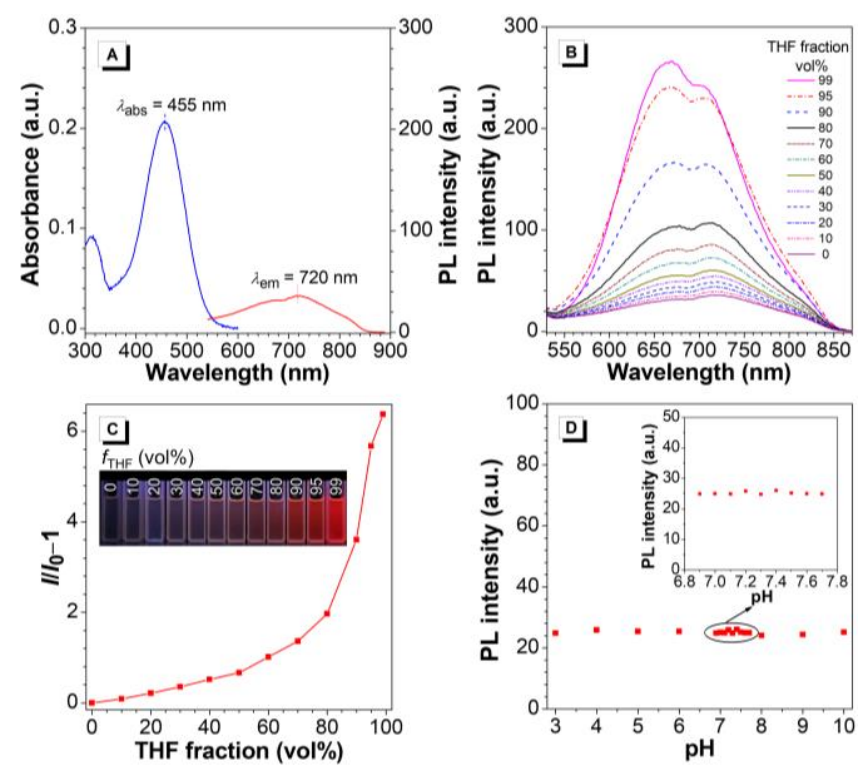

Figure 1. (A) Absorption $\left(c=10^{-5} \mathrm{M}\right)$ and emission spectra $\left(\lambda_{\mathrm{ex}}=455 \mathrm{~nm}, c=10^{-4} \mathrm{M}\right)$ of AIE-CNPy-AD in the DMSO solution at room temperature. (B) Emission spectra of AIE-CNPy-AD in the DMSO/THF mixtures with different THF fractions at room temperature, $\lambda_{\mathrm{ex}}=455 \mathrm{~nm}, c=10^{-5} \mathrm{M}$. (C) The plot of the emission enhancement $\left(I / I_{0}-1\right)$ versus the THF fractions. The inset: Corresponding fluorescent photographs of AIE-CNPy-AD in the DMSO/THF mixtures with different THF fractions taken under the irradiation at $365 \mathrm{~nm}$ with a handheld UV lamp.

(D) Maximum fluorescence intensity of AIE-CNPy-AD keeping unchanged under different pH values. 
Specific and High Affinity of AIE-CNPy-AD to A $\beta$ Fibrils. The binding affinity of AIE-CNPy-AD to hen egg white lysozyme (HEWL), a common model protein for amyloid studies, was evaluated first. As shown in Figure 2A, dramatic fluorescence enhancement at $620 \mathrm{~nm}$ was clearly observed with the increasing concentration of fibrilar HEWL in the PBS solution. In sharp contrast, AIE-CNPy-AD displayed much less marked fluorescence response to native HEWL at the same concentration level (Figure S6A). As compared to the emission in DMSO solution, the probe bound to HEWL showed an emission peak blue-shifted from 720 to $620 \mathrm{~nm}$, which might be attributed to the ICT effect and less polar microenvironment of the protein pockets. To our satisfaction, AIE-CNPy-AD has much higher ability to distinguish fibrilar HEWL from native ones, as ThT had the same response to fibrilar and native HEWL (Figures 2B, 2C, and Figure S6B). Moreover, due to the strong ACQ effect of ICG, just a small amount of fibrilar HEWL would prompt the quenching of fluorescence (Figure S6C and Figure 2C), making it unworkable in the detection of fibrilar HEWL. These results suggest that AIE-CNPy-AD possess higher possibility to function as a highly specific probe to $\mathrm{A} \beta$ fibrils in comparison to clinically used gold standard fluorescent probes such as ThT and ICG.

We then studied whether this AIE-active NIR probe could have a specific fluorescence response to $\mathrm{A} \beta$ fibrils. $\mathrm{A} \beta_{1-42}$ peptide was fully incubated to afford $\mathrm{A} \beta_{1-42}$ fibrils with expected fibrous or filamentous structure (Figure 2D). When the $\mathrm{A} \beta_{1-42}$ fibrils were added, emission intensity of AIE-CNPy-AD increased consistently with the emission peak blue-shifted to $620 \mathrm{~nm}$ like the situation of binding to HEWL (Figure 2E). Obviously, the background from AIE-CNPy-AD is far lower than that of ThT, which is merely 0.14 times that of ThT. Whereas, the signal of AIE-CNPy-AD upon interaction with $\mathrm{A} \beta_{1-42}$ fibrils is much higher than that of ThT under parallel conditions, which is about 1.4 times that of ThT (Figure S7). In consequence, the SNR of AIE-CNPy-AD reached 60 , which is 10 times to that of $\mathrm{ThT}\left(\mathrm{SNR}_{\mathrm{ThT}}=6\right.$; Figure $\left.2 \mathrm{~F}\right)$. The significantly enhanced SNR of AIE-CNPy-AD achieved by the minimization of background and amplification of signal could be interpreted as follows: (i) on one hand, the right hydrophilicity makes the AIE-CNPy-AD well dispersed in aqueous solution and the vigorous intramolecular motions efficiently exhaust the excited state, resulting in minimal emission; (ii) on the other hand, the strong binding of AIE-CNPy-AD to $\mathrm{A} \beta_{1-42}$ fibrils greatly hampered the intramolecular motions and activated the radiative decay channels, maximizing the fluorescence signal as a consequence 
of AIE effect. Ultrasensitive detection of $\mathrm{A} \beta_{1-42}$ fibrils could be expected.
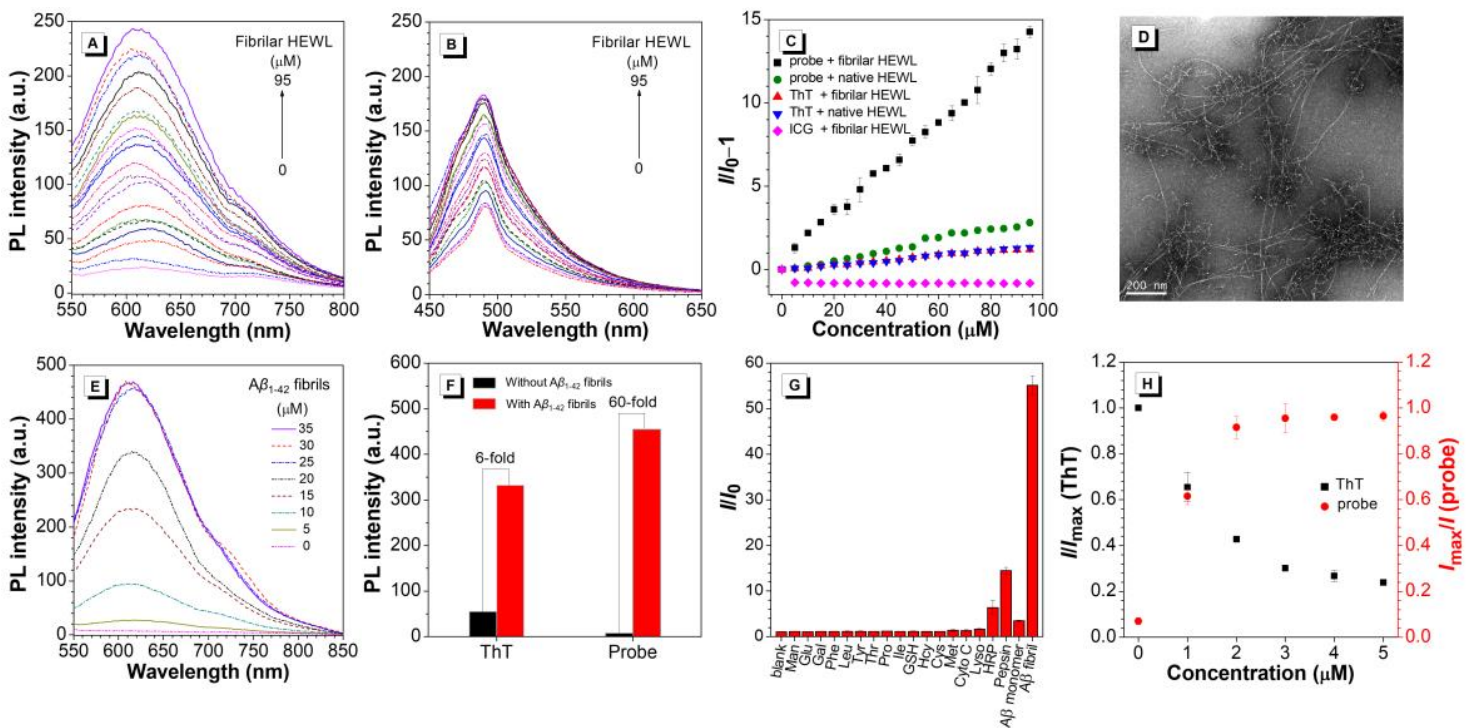

Figure 2. Emission spectra of (A) AIE-CNPy-AD $\left(\lambda_{\mathrm{ex}}=455 \mathrm{~nm}, c=5 \mu \mathrm{M}\right)$ and $(\mathrm{B})$ ThT $\left(\lambda_{\mathrm{ex}}=420 \mathrm{~nm}, c=5 \mu \mathrm{M}\right)$ in PBS solution $\left(\mathrm{pH}=7.4\right.$ ) with stepwise addition of fibrillar HEWL. (C) The plots of $I / I_{0}-1$ of probe AIE-CNPy-AD, ThT and ICG versus different concentrations of fibrilar HEWL and native HEWL, $c=5 \mu \mathrm{M}$. (D) TEM images of pre-formed $\mathrm{A} \beta 1-42$ fibrils. (E) Emission spectra of AIE-CNPy-AD in PBS solution (pH = 7.4) with stepwise addition of $\mathrm{A} \beta_{1-42}$ fibrils, $\lambda_{\mathrm{ex}}=455 \mathrm{~nm}, c=5 \mu \mathrm{M}$. (F) Fluorescence intensity of ThT and AIE-CNPy-AD bound with or without $\mathrm{A} \beta_{1-42}$ fibrils, respectively. (G) $I / I_{0}$ response assay versus various analytes. $(\mathrm{H})$ The plot of $I_{\max } / I$ of AIE-CNPy-AD and $I / I_{\max }$ of ThT versus different concentrations of AIE-CNPy-AD.

In addition to the ultra-high SNR, AIE-CNPy-AD also has high specificity to $\mathrm{A} \beta_{1-42}$ fibrils. A large variety of biological species including carbohydrates, amino acids, peptides, and other proteins were employed to assess specificity of AIE-CNPy-AD to $\mathrm{A} \beta_{1-42}$ fibrils (Figure $2 \mathrm{G}$, Figure S8). It is evident that AIE-CNPy-AD not only hardly has response to interfering small molecular species, but also has low response to potentially competitive peptides and enzymes with large molecular weight, especially $\mathrm{A} \beta_{1-42}$ monomer (Figure $2 \mathrm{G}$ and Figure $\mathrm{S} 8$ ).

Apart from specificity, binding affinity of the probe to analyte is also a vital parameter that ensures accurate tracing of the $\mathrm{A} \beta_{1-42}$ fibrils. Displacement assay of AIE-CNPy-AD against ThT-bound $\mathrm{A} \beta_{1-42}$ fibrils (Figure $\mathrm{S} 9$ and Figure $2 \mathrm{H}$ ) was then carried out to investigate the binding affinity. Fluorescence intensity of the pre-prepared ThT/A $\beta_{1-42}$ fibrils complex was firstly recorded with excitation at $420 \mathrm{~nm}$. AIE-CNPy-AD solution was subsequently added stepwise into the ThT/A $\beta_{1-42}$ complex, and the emission intensities of these two probes were measured under 
excitation at their corresponding maximum absorption wavelengths. It is observed that the emission intensity of ThT at $482 \mathrm{~nm}$ decreased continuously with increasing concentration of AIE-CNPy-AD; and in the meantime, fluorescence of AIE-CNPy-AD peaked at $620 \mathrm{~nm}$ emerged and was enhanced accordingly. Remarkably, it is indicated that AIE-CNPy-AD displaced ThT from ThT/A $\beta$ fibrils complex to generate the more strongly bound AIE-CNPy-AD/A $\beta$ fibrils complex in the solution. Besides, dissociation constant $\left(K_{\mathrm{d}}\right)^{41}$ of AIE-CNPy-AD was calculated to be $185 \mathrm{nM}$ (Figure S10), considerably smaller than that of ThT $(890 \mathrm{nM})^{42}$. Sufficient evidences proved that AIE-CNPy-AD has much higher binding affinity to $\mathrm{A} \beta_{1-42}$ fibrils than ThT.

\section{High-Contrast and High-Resolution In-Vitro Fluorescent Staining of Paraffin}

Slices of Mice Brain. It is confirmed that AIE-CNPy-AD, with high specificity, binding affinity and SNR, exhibits excellent performance on precise detection of $\mathrm{A} \beta_{1-42}$ fibrils in solution. To explore the ability of AIE-CNPy-AD labelling $\mathrm{A} \beta$ plaques in brain tissues, in-vitro fluorescent staining of paraffin mice brain slices resected from $5 * \mathrm{FAD}$ transgenic mice, APP/PS1 transgenic mice and age-matched wild-type mice was conducted with AIE-CNPy-AD. False signals originated from the binding of AIE-CNPy-AD or antibody with proteins or interfering species in brain cells could be identified through the localization of nuclei with Hoechst 33342. Notably, as displayed in Figure 3 and Figure S11, no matter in 2.5-month-old 5*FAD transgenic mice or in 6-month-old APP/PS1 transgenic mice, $\mathrm{A} \beta$ plaques were unambiguously visualized with bright red fluorescence. Specific labeling of $\mathrm{A} \beta$ plaques with high contrast and high resolution in these mice indicates that AIE-CNPy-AD is universal to label $\mathrm{A} \beta$ plaques in different strains of mice. ${ }^{43,44}$ Moreover, it can be easily seen from the CLSM images, the $\mathrm{A} \beta$ plaques in 2.5 -month-old $5 * \mathrm{FAD}$ transgenic mice (Figure 3A and Figure S11A-S11C) are larger and more densely distributed than that in 6-month-old APP/PS1 transgenic mice (Figure 3F and Figure S11D-S11F). The experimental results agreed well with situation of early plaque formation at the time point in these two strains of mice, implying the reliability of AIE-CNPy-AD in fluorescent staining of $\mathrm{A} \beta$ plaques in slices of mice brain. To our delight, there was no observable intracell fluorescence signal, which implied that our probe is preferentially bound to the $\mathrm{A} \beta$ plaques generally forming extracellularly, eliminating the false signal from intracellular species. Simultaneously, the signals in the red channel agree fairly well with those in the green channel manifested that AIE-CNPy-AD has extremely high specificity comparable to that of antibody, to $\mathrm{A} \beta$ plaques (Figure $3 \mathrm{~B}-3 \mathrm{E}$, 
3G-3J). In contrast with transgenic mice, no plaques were found in the age-matched wild-type mice no matter observing from red or green channel (Figure S12), which not only verified the high specificity of AIE-CNPy-AD to $\mathrm{A} \beta$ plaques for a second time, but also suggested the super-high fidelity of the designed probe in real brain tissues.
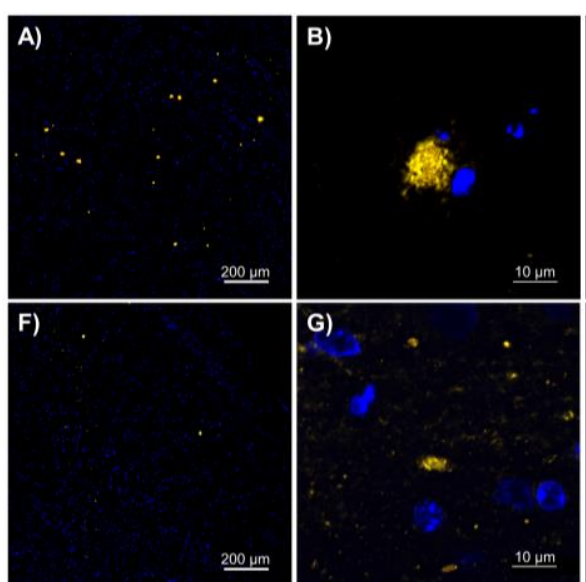

$200 \mu \mathrm{m}$

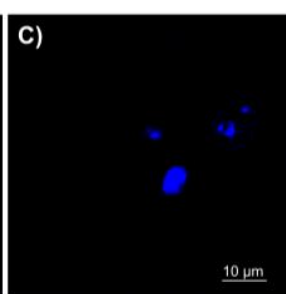

H)
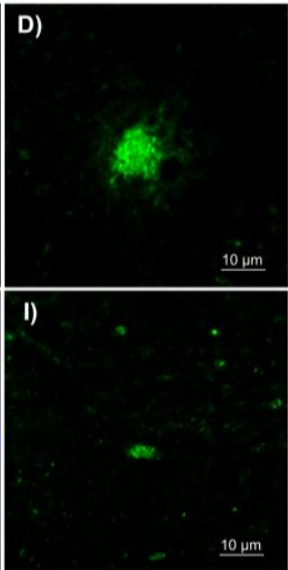

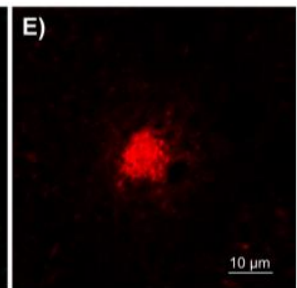

J)

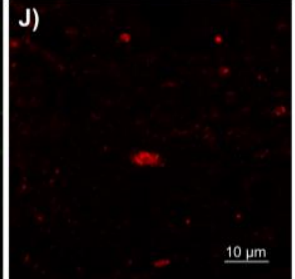

Figure 3. Fluorescent staining of the brain slices of (A-E) 2.5-month-old $5 * \mathrm{FAD}$ transgenic mice and (F-J) 6-month-old APP/PS1 transgenic mice. The slices were co-stained with AIE-CNPy-AD, rabbit anti-mouse primary antibody (anti-beta amyloid 1-42 (mOC64), i.e., ab201060), Alexa Fluor® 488-labeled goat anti-rabbit secondary antibody (ab150077), and Hoechst 33342 in sequence. (A, B, F, G) Merged images of three channels; (C, H) Blue channel: Hoechst 33342, $\lambda_{\mathrm{ex}}=405 \mathrm{~nm}, \lambda_{\mathrm{em}}=461 \mathrm{~nm}$; (D, I) Green channel: Alexa Fluor ${ }^{\circledR} 488, \lambda_{\mathrm{ex}}=488 \mathrm{~nm}, \lambda_{\mathrm{em}}=$ $519 \mathrm{~nm}$; (E, J) Red channel: AIE-CNPy-AD, $\lambda_{\mathrm{ex}}=488 \mathrm{~nm}, \lambda_{\mathrm{em}}=620 \mathrm{~nm} .(\mathrm{A}, \mathrm{F}) 100 \times$ magnification, $(\mathrm{B}-\mathrm{E}, \mathrm{G}-\mathrm{J})$ $2000 \times$ magnification.

\section{In-Vivo Imaging of $\mathrm{A} \beta$ plaques in Live Mice with Outstanding BBB penetrability.}

Inspired by the superb red fluorescence light-up $\mathrm{A} \beta$ plaque-specific response in the brain slices of mice, we further evaluated the biocompatibility and BBB penetrability of AIE-CNPy-AD which is indispensable before in-vivo imaging. Cell viability experiments were carried out to ascertain the biocompatibility of AIE-CNPy-AD by CCK-8 assays. Mouse breast cancer cells (4T1) and human breast cancer cells (MCF-7) were incubated with different concentrations of AIE-CNPy-AD for 24 $\mathrm{h}$, respectively. The cell viabilities of two cell lines both kept at a level close to $100 \%$ even at a probe concentration of $32 \mu \mathrm{M}$ (Figure S13A and Figure S13B). Besides, MCF-7 cells were chosen as an example to be incubated with different concentrations of AIE-CNPy-AD for $48 \mathrm{~h}$ and all the cell viabilities were still higher than $95 \%$ (Figure S13C). The results provided strong evidence to the fact that AIE-CNPy-AD has low cell cytotoxicity and favorable biocompatibility to various 
cells, demonstrating its high applicability to live animals. The oil-water partition coefficient $(\log P)$ is often used as an index indicating the possible ability of penetrating $B B B .{ }^{45}$ The $\log P$ of AIE-CNPy-AD was determined to be 1.24 by shaking-flask method, which is far larger than that of ThT $(0.16),{ }^{8}$ suggestive of the higher lipophilicity and BBB penetrating potential of AIE-CNPy-AD as compared with ThT.

The feasibility of AIE-CNPy-AD tracking $\mathrm{A} \beta$ plaques in vivo was confirmed using 2.5-month-old 5*FAD mice, 6-month-old APP/PS1 mice and age-matched wild-type mice as model mice. Live imaging of these mice was performed after the tail vein injection of AIE-CNPy-AD (Figure 4). Almost all the fluorescence signals were clearly witnessed in the center of brain compartments and able to be efficiently captured. Apparently, the fluorescence signals in the brain regions of 2.5 -month-old $5 *$ FAD mice (Figure 4A) were already much stronger than those in the wild-type mice at 5 min post injection (Figure 4B). Particularly, with the decay of signal, difference in signal intensity recorded from $5 * \mathrm{FAD}$ and wild-type mice was enlarged as indicated by the semi-quantitative analysis of the images (Figure 4E). Similarly, intense fluorescence signal is readily visible from the brain area of 6-month-old APP/PS1 mice after the injection of AIE-CNPy-AD. Moreover, the contrast between the fluorescence signals from APP/PS1 and 6-month-old wild-type mice is quite dramatic. Compared with $5 *$ FAD mice, difference in the signal intensity between APP/PS1 mice and age-matched wild-type mice was 4.7 times larger than that between $5 * \mathrm{FAD}$ mice and the corresponding wild-type mice at 5 min after being injected with AIE-CNPy-AD (Figure 4G, 4H, and 4K). It might be because the formation of $\mathrm{A} \beta$ deposits in APP/PS1 mice is slower than that in $5^{*} \mathrm{FAD}$ mice, which probably gives more $\mathrm{A} \beta$ oligomers in the brain region. Thus, partial of the AIE-CNPy-AD molecules maybe bind to $\mathrm{A} \beta$ oligomer, causing the obvious differences in the fluorescence signal between these two mouse strains. In addition, fluorescence signal intensity of APP/PS1 mice declined faster than that of 5*FAD mice after being injected with AIE-CNPy-AD for $30 \mathrm{~min}$, as it can be seen from the semi-quantitative analysis of the images (Figure 4K). It is possibly because that there are larger and more $\mathrm{A} \beta$ plaques in 2.5-month-old 5*FAD mice than in 6-month-old APP/PS1 mice, which slows down the clearance of AIE-CNPy-AD from the brain. Notably, whether in $5 *$ FAD mice or APP/PS1 mice at $1 \mathrm{~h}$ post probe injection, $F(\mathrm{t}) / F($ Pre $)$ value of transgenic mice was at least 1.75 times that of wild-type mice, indicative of the potential of AIE-CNPy-AD to realize long-term 
tracking of $\mathrm{A} \beta$ plaques in vivo.

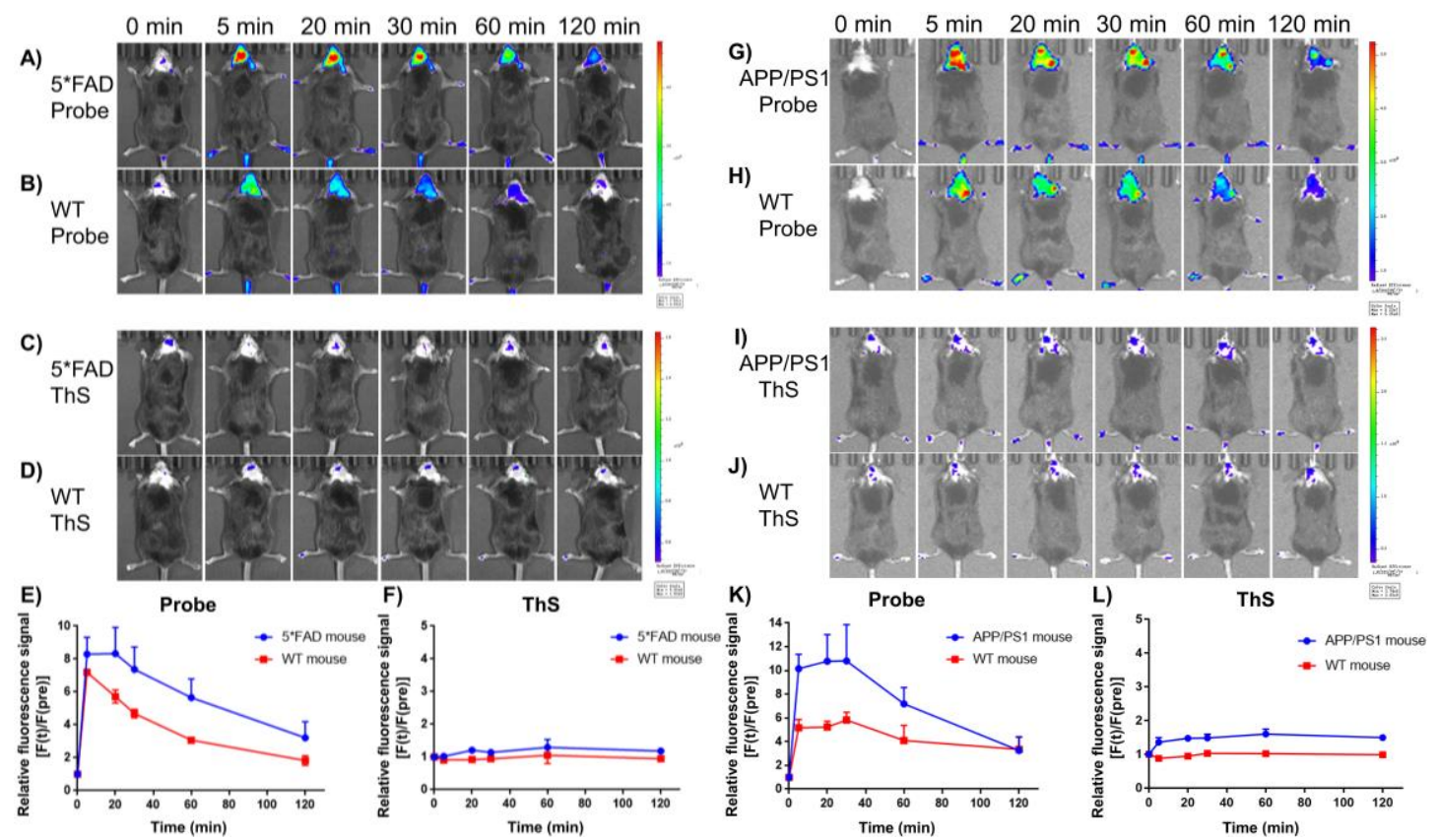

Figure 4. Fluorescence images of (A) $5 *$ FAD mice (2.5-month-old) and (B) wild-type mice (2.5-month-old) at different time points before and after via tail vein injection of AIE-CNPy-AD ( $2 \mathrm{mg} / \mathrm{kg})$. Fluorescence images of (C) $5 *$ FAD mice (2.5-month-old) and (D) wild-type mice (2.5-month-old) at different time points before and after via tail vein injection of $\mathrm{ThS}(2 \mathrm{mg} / \mathrm{kg})$. The relative fluorescence signal $[F(\mathrm{t}) / F($ pre $)]$ in the brain regions of $5 * \mathrm{FAD}$ mice and wild-type mice after via tail vein injection of (E) AIE-CNPy-AD (2 mg/kg) or (F) ThS (2 mg/kg). Fluorescence images of (G) APP/PS1 mice (6-month-old) and (H) wild-type mice (6-month-old) at different time points before and after via tail vein injection of AIE-CNPy-AD (2 mg/kg). Fluorescence images of (I) APP/PS1 mice (6-month-old) and (J) wild-type mice (6-month-old) at different time points before and after via tail vein injection of ThS $(2 \mathrm{mg} / \mathrm{kg})$. The relative fluorescence signal $[F(\mathrm{t}) / F(\mathrm{pre})]$ in the brain regions of APP/PS1 mice and wild-type mice after via tail vein injection of (K) AIE-CNPy-AD (2 mg/kg) or (L) ThS (2 mg/kg). AIE-CNPy-AD: $\lambda_{\mathrm{ex}}=500 \mathrm{~nm}, \lambda_{\mathrm{em}}=620 \mathrm{~nm} ; \mathrm{ThS}: \lambda_{\mathrm{ex}}=430 \mathrm{~nm}, \lambda_{\mathrm{em}}=500 \mathrm{~nm}$.

The case as for ThS is very different from that of AIE-CNPy-AD. As shown in Figure 4C, 4D, 4F, 4I, 4J, and 4L, hardly any valid signal was found from the 5*FAD mice, APP/PS1 mice and wild-type mice administrated with $\mathrm{ThS}$, clearly revealing the powerlessness of $\mathrm{ThS}$ in in-vivo imaging of $\mathrm{A} \beta$ plaques. This is most probably the result of the combination of its poor blood-brain barrier (BBB) permeability, short excitation and emission wavelengths which cannot penetrate the skull of mice, and the ACQ effect. ${ }^{46}$ Undoubtedly, these visualization results directly validated that AIE-CNPy-AD is capable of penetrating the $\mathrm{BBB}$ and image $\mathrm{A} \beta$ fibrils/plaques in vivo with 
ultra-high contrast and fidelity.

In-Vivo Tracking of A $\beta$ Plaques in APP/PS1 Mice at A Super-Early Stage. It has confirmed that AIE-CNPy-AD exhibits significant signal difference between transgenic mice and wild-type mice in a relatively long time-period post probe administration. It can be envisaged that AIE-CNPy-AD might be promising for early diagnosis of $\mathrm{AD}$ in transgenic mice by virtue of the A $\beta$ plaques-sepecific in-vivo imaging ability of AIE-CNPy-AD. Young APP/PS1 transgenic mice of 2-month-, 3-month-, 4-month-, and 6-month-old and age-matched wild-type (WT) mice were thus employed to systematically assess the performance of AIE-CNPy-AD in early diagnosis of $\mathrm{AD}$ (Figure 5 and Figure S14). It was observed that the difference in the fluorescence signal intensity of APP/PS1 transgenic mice and wild-type mice already became very evident at the age of 4 month. In the meantime, the fluorescence signal intensity is positively correlated with the age of transgenic $\mathrm{AD}$ mice (Figure 5A). More specifically, at 20 min post injection, the $F(\mathrm{t}) / F(\operatorname{Pre})$ value of the 4-month-old APP/PS1 transgenic mice was 1.86 times that of the age-matched WT mice, and the $F(\mathrm{t}) / F(\mathrm{Pre})$ value of 6-month-old APP/PS1 transgenic mice was 2.06 times that of the age-matched WT mice (Figure 5B). Meanwhile, there was no apparent signal difference in WT mice of different months (Figure 5C), and the $F(\mathrm{t}) / F(\operatorname{Pre})$ values of 2-month-old and 3-month-old APP/PS1 transgenic mice were almost the same as those of the age-matched WT mice (Figure 5D). These imaging results especially those acquired with APP/PS1 transgenic mice at an age of 4-month-old clearly showed that AIE-CNPy-AD is competent for the precise diagnosis of AD at a super-stage. It has been speculated that the reason for the failure of early drug intervention on $\mathrm{AD}$ is probably that intervention in the phase is not early enough, which in turn is greatly related to the efficient capture of biological manifestations rather than clinical manifestations in the early diagnosis. ${ }^{47,48}$ The experimental results obtained with AIE-CNPy-AD is of great significance because APP/PS1 transgenic mice are found to exhibit memory deficits from 5 months old on which is early clinical presentations, confirming that AIE-CNPy-AD can diagnose AD of APP/PS1 transgenic mice during the period of early biological manifestations, prior to the appearance of clinical presentations. ${ }^{43,47,49}$ Furthermore, as shown in Figure S15, ThS could neither give discriminatory signals between the APP/PS1 transgenic mice and wild-type mice nor provide distinct signals among mice of different ages, manifesting that our probe outperforms the clinically used gold-standard probe and could be used as an upgraded alternative to the 
commercially available ones.
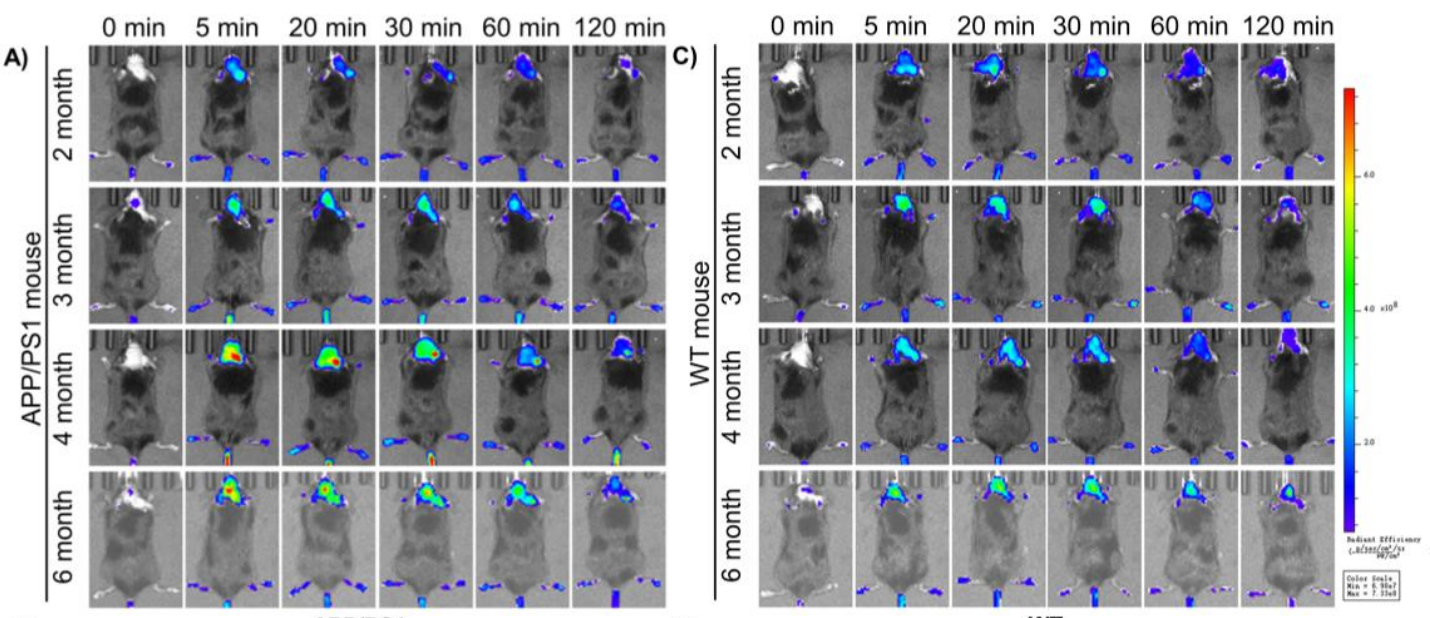

B)

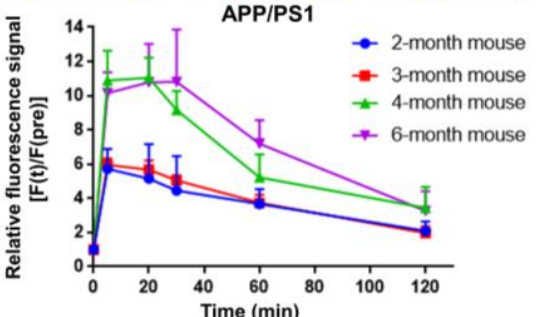

D)

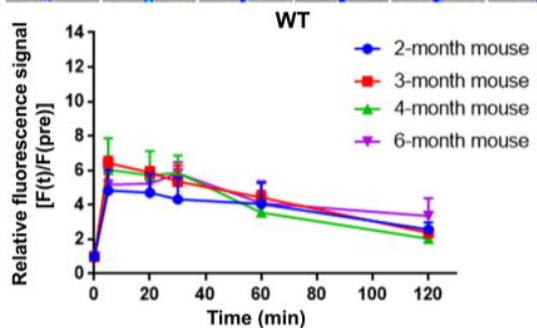

Figure 5. Fluorescence images of (A) APP/PS1 mice or (C) wild-type mice of different ages (2-month-old, 3-month-old, 4-month-old, 6-month-old) at different time points taken before or at different time points after tail vein injection of AIE-CNPy-AD (2 mg/kg). The relative fluorescence signal $[F(\mathrm{t}) / F(\mathrm{pre})]$ in the brain regions of $(\mathrm{B})$ APP/PS1 mice or (D) wild-type mice of different ages after via tail vein injection of AIE-CNPy-AD (2 mg/kg). AIE-CNPy-AD: $\lambda_{\mathrm{ex}}=500 \mathrm{~nm}, \lambda_{\mathrm{em}}=620 \mathrm{~nm}$.

\section{Ex-Vivo Fluorescent Staining of Frozen Slices of Mice Brain Administrated} Probe Further Validate Its High Fidelity of A $\boldsymbol{\beta}$ Plaques-Specific Imaging. To further examine whether the AIE-CNPy-AD binds exclusively to $\mathrm{A} \beta$ plaques in the brain of live mice, the APP/PS1 transgenic mice of different ages and age-matched wild-type mice were injected with AIE-CNPy-AD and sacrificed at 15 min post-administration. The frozen slices of these mice brain were then stained with rabbit anti-mouse primary antibody (ab201060), Alexa Fluor® 488-labeled goat anti-rabbit secondary antibody (ab150077), and Hoechst 33342 in sequence. As shown in Figure 6A, 6F, and Figure S16, the $\mathrm{A} \beta$ plaques were imaged with high resolution and high contrast. Moreover, it can be clearly observed that the $\mathrm{A} \beta$ plaques in brain slices of 6-month-old APP/PS1 transgenic mice were larger and richer in number than those of 4-month-old APP/PS1 transgenic mic, while no significant A $\beta$ plaques were found in the brain slices of 2-month-old, 3-month-old APP/PS1 transgenic mice (Figure S16), and all the 
age-matched WT mice (Figure S17). Manifestly, AIE-CNPy-AD indeed labelled A $\beta$ plaques in vivo with ultrahigh fidelity and specificity, as suggested by the very good overlap between the green (antibody) and red channels (AIE-CNPy-AD; Figure 6B-6E and 6G-6J).

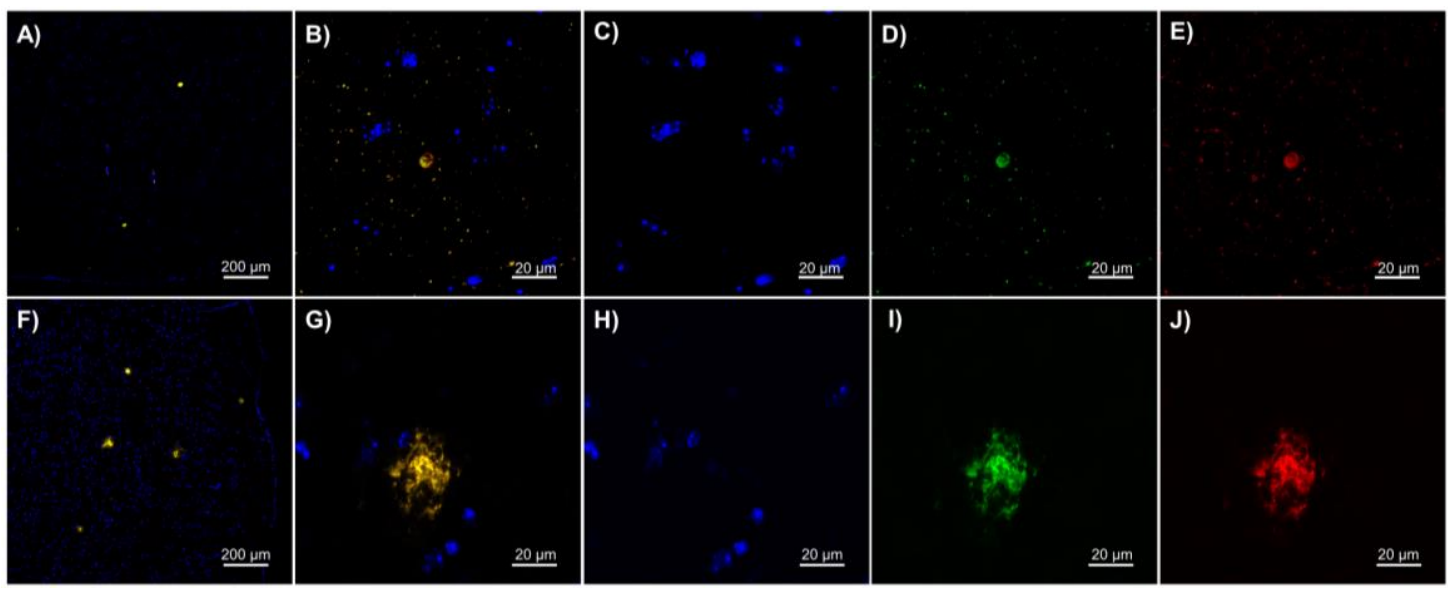

Figure 6. Fluorescent staining of the brain slices of (A-E) 4-month-old APP/PS1 transgenic AD mice and (F-J) 6-month-old APP/PS1 transgenic AD mice. The slices of mice were pre-injected with AIE-CNPy-AD via tail vein injection, and then sequentially stained with anti-beta amyloid 1-42 (mOC64; ab201060) and Alexa Fluor® 488-labeled goat anti-rabbit secondary antibody (ab150077) after sacrificing. (A, B, F, G) Merged image of three channels; (C, H) Blue channel: Hoechst 33342, $\lambda_{\mathrm{ex}}=405 \mathrm{~nm}, \lambda_{\mathrm{em}}=461 \mathrm{~nm}$; (D, I) Green channel: Alexa Fluor ${ }^{\circledR}$ 488-labeled antibody, $\lambda_{\mathrm{ex}}=488 \mathrm{~nm}, \lambda_{\mathrm{em}}=519 \mathrm{~nm}$; (E, J) Red channel: AIE-CNPy-AD, $\lambda_{\mathrm{ex}}=488 \mathrm{~nm}, \lambda_{\mathrm{em}}=620 \mathrm{~nm}$. (A, F) 100× magnification; (B-E, G-J) 1000× magnification.

Elucidation of the Working Mechanism with Molecular Docking Simulations. We believe that the rod-like architecture, extended D- $\pi$-A electronic structure, flexible 3D conformation, the amphiphilic and zwitterionic molecular structure collectively contribute to the outstanding performance of AIE-CNPy-AD in $\mathrm{A} \beta$ fibrils/plaques-specific detection and imaging. As above mentioned, the rod-like structure is supposed to favors the recognition of $\beta$ sheets. Moreover, the hydrophobic $\pi$-conjugated backbone and the pyridyl and sulfonate groups are envisaged to benefit the binding of AIE-CNPy-AD to $\mathrm{A} \beta$ species possibly via hydrophobic interaction, $\pi-\pi$ interaction, and electrostatic interaction. When in solution or molecularly dispersed, the AIE-CNPy-AD molecules undergo active intramolecular motions, leading to an OFF state and a low background. When coexists with $\mathrm{A} \beta$ monomer, intramolecular motions of AIE-CNPy-AD are merely weakly restricted, rendering weak red emission released (near-OFF state), due to the weak binding affinity of AIE-CNPy-AD and $\mathrm{A} \beta$ monomer. On the contrary, 
strong interactions between AIE-CNPy-AD and fibrils impose severe restriction on the intramolecular motions, which activates the AIE process, ${ }^{50,51}$ and switches AIE-CNPy-AD from OFF to ON state to emit strong red/NIR fluorescence. Thus, AIE-CNPy-AD can distinguish $\mathrm{A} \beta$ fibrils from $\mathrm{A} \beta$ monomers precisely (Figure 7A).

A)

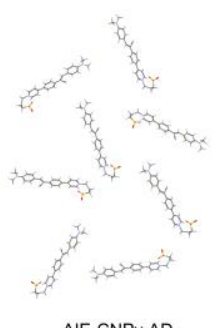

Miscible in aqueous media Active intramolecular motions Non-emission Non-emission
OFF state, low background
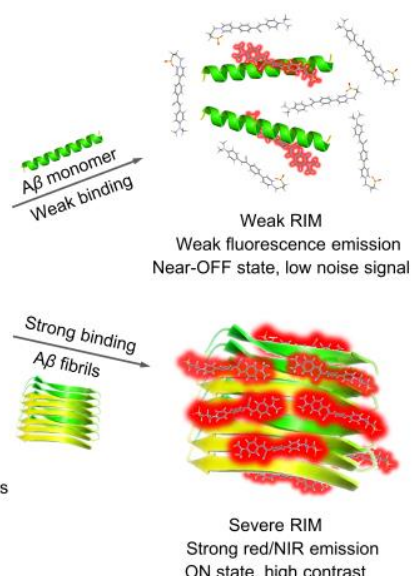

B)

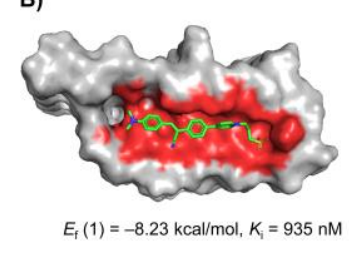

D)

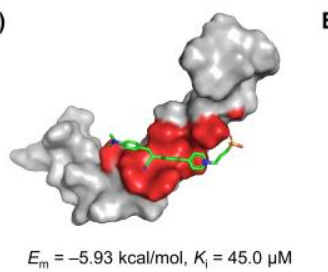

C) Short contacts (A)

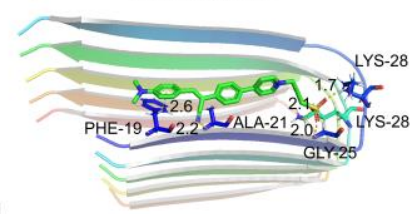

E) Short contacts (A)

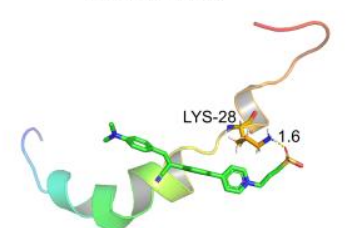

Figure 7. (A) Possible working mechanism of AIE-CNPy-AD in the specific detection of A $\beta$ fibrils. The lowest energy binding mode of AIE-CNPy-AD with A $\beta$ fibrils (PDB ID: 2BEG) in (B) surface representation ${ }^{52}$ and (C) cartoon representation. The lowest energy binding mode of AIE-CNPy-AD with A $\beta$ monomer (PDB ID: 1 Z0Q) in (D) surface representation and (E) cartoon representation. $E_{\mathrm{f}}$ : binding energy of AIE-CNPy-AD with A $\beta$ fibrils; $E_{\mathrm{m}}$ : binding energy of AIE-CNPy-AD with $\mathrm{A} \beta$ monomer. $K_{\mathrm{i}}$ is the inhibition constant.

Molecular docking simulations were carried out to unveil the interactions between AIE-CNPy-AD and $\mathrm{A} \beta$ fibrils or monomer and to elucidate the working mechanism of specific detection of $\mathrm{A} \beta$ fibrils. As revealed by the three top docking conformations with the lowest binding free energies shown in Figure 7B, 7C and Figure S18, the most preferable binding direction of AIE-CNPy-AD to $\mathrm{A} \beta$ fibrils is consistent with the orientation of the $\beta$-sheets of $\mathrm{A} \beta$ fibrils, which proves that rod-shaped geometric structure of AIE-CNPy-AD is very helpful to the binding with $\mathrm{A} \beta$ fibrils. ${ }^{53}$ Simultaneously, the hydrophobic PHE-19 residue on the $\beta$-sheets of $\mathrm{A} \beta$ fibrils has strong $\mathrm{C}-\mathrm{H} \cdots \pi$ interaction $(2.6 \AA)$ with the phenyl ring of AIE-CNPy-AD, and the ALA-21 residue exhibits very strong interaction with the cyano group of AIE-CNPy-AD (2.2 A ). To our astonishment, the GLY-25 residue and two LYS-28 residues have strong hydrogen bonding interactions with the sulfonate group of AIE-CNPy-AD (1.7-2.1 $\AA$ ). It is obvious that sulfonate not only improves water solubility of AIE-CNPy-AD, but also further enhances the binding ability of AIE-CNPy-AD to $\mathrm{A} \beta$ fibrils via H-bonding and electrostatic interactions. Collectively, the 
strong intermolecular interactions between AIE-CNPy-AD and $\mathrm{A} \beta$ fibrils greatly hinder the intramolecular motions of AIE-CNPy-AD to give out the "lighted-up" fluorescent response. ${ }^{54}$

In contrast, only the LYS- 28 residue of $\mathrm{A} \beta$ monomer shows hydrogen bonding interaction with the sulfonate unit of AIE-CNPy-AD and no other interactions exist, which results in weaker restriction on the intramolecular motions and weak fluorescence (Figure 7D and Figure 7E). The inhibition constant ${ }^{55}\left(K_{\mathrm{i}}\right)$ of AIE-CNPy-AD and $\mathrm{A} \beta$ fibrils $(935 \mathrm{nM})$ is far smaller than that of AIE-CNPy-AD and $\mathrm{A} \beta$ monomer $(45.0 \mu \mathrm{M})$. Simultaneously, the lowest docking energy of AIE-CNPy-AD and $\mathrm{A} \beta$ fibrils was calculated to be $-8.23 \mathrm{kcal} / \mathrm{mol}$, which is substantially lower than that of AIE-CNPy-AD and A $\beta$ monomer $(-5.93 \mathrm{kcal} / \mathrm{mol})$. As exhibited in Table $\mathrm{S} 1$, all the ten best binding poses with the lowest energies of AIE-CNPy-AD and $\mathrm{A} \beta$ fibrils consistently display lower docking energies than those of AIE-CNPy-AD and $\mathrm{A} \beta$ fibrils. These data sufficiently suggested that binding affinity of AIE-CNPy-AD to $\mathrm{A} \beta$ fibrils is much stronger than that of AIE-CNPy-AD to $\mathrm{A} \beta$ monomer. Moreover, the binding energy of AIE-CNPy-AD and $\mathrm{A} \beta$ fibrils is lower than that of ThT and $\mathrm{A} \beta$ fibrils $(-7.18 \mathrm{kcal} / \mathrm{mol}),{ }^{56}$ indicating that the AIE-CNPy-AD possesses higher affinity to $\mathrm{A} \beta$ fibrils as compared to ThT. The molecular docking simulation results are powerful proofs to our experiment results and further verify the rationality and feasibility of our design strategy.

\section{Conclusion}

Precise in-vivo tracking of $\mathrm{A} \beta$ fibrils/plaques is of great significance in both fundamental research and technological development. In this work, we proposed an 'all-in-one' molecular design strategy and implemented it in the development of a novel AIE-active NIR-emissive probe (AIE-CNPy-AD) for specifically discriminating $\mathrm{A} \beta$ fibrils and imaging $\mathrm{A} \beta$ plaques in vivo at an early stage. Rod-shaped amphipathic AIE-CNPy-AD possesses excellent BBB penetrability and high binding affinity to $\mathrm{A} \beta$ fibrils. The $\mathrm{D}-\pi-\mathrm{A}$ electronic structure with multiple rotors and flexible $3 \mathrm{D}$ conformations endue AIE-CNPy-AD with red/NIR emission, AIE feature, and resultant excellent tracing capability of $\mathrm{A} \beta$ fibrils in vivo with superior tissue penetration ability, ultra-high signal-to-noise ratio and high fidelity. More importantly, our probe also enjoys the following advantages: (1) the raw materials are cheap and easily available, and the synthesis is fairly simple; (2) the in-vivo imaging capability is universal and not restricted by the strain of mouse; (3) the 
precise in-vivo tracking of $\mathrm{A} \beta$ fibrils could be realized at a super-early stage before the occurrence of clinical manifestations. Moreover, the rationality and feasibility were fully verified and the working mechanism of AIE-CNPy-AD was elucidated by the molecular docking simulation results. The present work will not only shed light on the rational design of high-performance fluorescent probes for accurately imaging of $\mathrm{A} \beta$ fibrils in vivo, but also provide a promising diagnostic tool to diagnose $\mathrm{AD}$ at an early stage and find timing of early drug intervention.

\section{Notes}

The authors declare no competing financial interest.

\section{Acknowledgements}

We are grateful for the financial support from the NSFC/China (Nos. 21788102, 21875064, 81903545, 21604023, and 21790361), Shanghai Municipal Science and Technology Major Project (No. 2018SHZDZX03), Programme of Introducing Talents of Discipline to Universities (No. B16017), Shanghai Science and Technology Committee (No. 17520750100), Beijing New-Star Plan of Science and Technology (No. Z201100006820009), the Shanghai Sailing Program (No. 16YF1402200), and the Fundamental Research Funds for the Central Universities.

\section{Reference}

(1) Yang, J.; Zeng, F.; Ge, Y.; Peng, K.; Li, X.; Li, Y.; Xu, Y. Development of Near-Infrared Fluorescent Probes for Use in Alzheimer's Disease Diagnosis. Bioconjug. Chem. 2020, 31, 2-15.

(2) Aliyan, A.; Cook, N.; Marti, A. Interrogating Amyloid Aggregates using Fluorescent Probes. Chem. Rev. 2019, 119, 11819-11856.

(3) Zhou, K.; Bai, H.; Feng, L.; Dai, J.; Cui, M. Smart D- $\pi$-A Type Near-Infrared A $\beta$ Probes: Effects of a Marked $\pi$ Bridge on Optical and Biological Properties. Anal. Chem. 2017, 89 9432-9437.

(4) Li, Y.; Xu, D.; Ho, S. L.; Li, H. W.; Yang, R.; Wong, M. S. A Theranostic Agent for In Vivo Near-Infrared Imaging of $\beta$-Amyloid Species and Inhibition of $\beta$-Amyloid Aggregation. Biomaterials 2016, 94, 84-92.

(5) Cai, J.; Yi, P.; Miao, Y.; Liu, J.; Hu, Y.; Liu, Q.; Feng, Y.; Chen, H.; Li, L. Ultrasmall T1-T2 Magnetic Resonance Multimodal Imaging Nanoprobes for the Detection of $\beta$-Amyloid Aggregates in Alzheimer's Disease Mice. ACS Appl. Mater. Interfaces 2020, 12, 26812-26821. 
(6) Fernandez, T.; Martinez-Serrano, A.; Cusso, L.; Desco, M.; Ramos-Gomez, M. Functionalization and Characterization of Magnetic Nanoparticles for the Detection of Ferritin Accumulation in Alzheimer's Disease. ACS Chem. Neurosci. 2018, 9, 912-924.

(7) Cui, M.; Ono, M.; Watanabe, H.; Kimura, H.; Liu, B.; Saji, H. Smart Near-infrared Fluorescence Probes with Donor-Acceptor Structure for In Vivo Detection of $\beta$-Amyloid Deposits. J. Am. Chem. Soc. 2014, 136, 3388-3394.

(8) Fu, W.; Yan, C.; Guo, Z.; Zhang, J.; Zhang, H.; Tian, H.; Zhu, W. H. Rational Design of Near-Infrared Aggregation-Induced-Emission-Active Probes: In Situ Mapping of Amyloid- $\beta$ Plaques with Ultrasensitivity and High-Fidelity. J. Am. Chem. Soc. 2019, 141, 3171-3177.

(9) Wang, J.; Zhang, Z.; Zhang, H.; Li, C.; Chen, M.; Liu, L.; Dong, M. Enhanced Photoresponsive Graphene Oxide-Modified $g-\mathrm{C}_{3} \mathrm{~N}_{4}$ for Disassembly of Amyloid $\beta$ Fibrils. ACS Appl. Mater. Interfaces 2019, 11, 96-103.

(10) Xia, N.; Zhou, B.; Huang, N.; Jiang, M.; Zhang, J.; Liu, L. Visual and Fluorescent Assays for Selective Detection of $\beta$-Amyloid Oligomers Based on the Inner Filter Effect of Gold Nanoparticles on the Fluorescence of CdTe Quantum Dots. Biosens. Bioelectron. 2016, 85, $625-632$.

(11) Fu, H.; Tu, P.; Zhao, L.; Dai, J.; Liu, B.; Cui, M. Amyloid- $\beta$ Deposits Target Efficient Near-Infrared Fluorescent Probes: Synthesis, In Vitro Evaluation, and In Vivo Imaging. Anal. Chem. 2016, 88, 1944-1950.

(12) Li, Y.; Xu, D.; Sun, A.; Ho, S. L.; Poon, C. Y.; Chan, H. N.; Ng, O. T. W.; Yung, K. K. L.; Yan, H.; Li, H. W.; Wong, M. S. Fluoro-Substituted Cyanine for Reliable in vivo Labelling of Amyloid- $\beta$ Oligomers and Neuroprotection against Amyloid- $\beta$ Induced Toxicity. Chem. Sci. 2017, 8, 8279-8284.

(13) Heo, C. H.; Sarkar, A. R.; Baik, S. H.; Jung, T. S.; Kim, J. J.; Kang, H.; Mook-Jung, I.; Kim, H. M. A Quadrupolar Two-photon Fluorescent Probe for In Vivo Imaging of Amyloid- $\beta$ Plaques. Chem. Sci. 2016, 7, 4600-4606.

(14) Peng, C.; Wang, X.; Li, Y.; Li, H. W.; Wong, M. S. Versatile Fluorescent Probes for Near-infrared Imaging of Amyloid- $\beta$ Species in Alzheimer's Disease Mouse Model. J. Mater. Chem. B 2019, 7, 1986-1995. 
(15) Wang, Y. L.; Fan, C.; Xin, B.; Zhang, J. P.; Luo, T.; Chen, Z. Q.; Zhou, Q. Y.; Yu, Q.; Li, X. N.; Huang, Z. L.; Li, C.; Zhu, M. Q.; Tang, B. Z. AIE-Based Super-Resolution Imaging Probes for $\beta$-Amyloid Plaques in Mouse Brains. Mater. Chem. Front. 2018, 2, 1554-1562.

(16) Dou, W. T.; Zhang, J. J.; Li, Q.; Guo, Z.; Zhu, W.; Chen, G. R.; Zhang, H. Y.; He, X. P. Fluorescence Imaging of Alzheimer's Disease with a Flat Ensemble Formed between a Quinoline-Malononitrile AIEgen and Thin-Layer Molybdenum Disulfide. ChemBioChem 2019, 20, $1856-1860$.

(17) Zhao, Y.; Li, X.; Yang, Y.; Si, S.; Deng, C.; Wu, H. A Simple Aptasensor for A $\beta_{40}$ Oligomers Based on Tunable Mismatched Base Pairs of dsDNA and Graphene Oxide. Biosens. Bioelectron. 2020, $149,111840$.

(18) Zhou, K.; Li, Y.; Peng, Y.; Cui, X.; Dai, J.; Cui, M. Structure-Property Relationships of Polyethylene Glycol Modified Fluorophore as Near-Infrared A $\beta$ Imaging Probes. Anal. Chem. 2018, $90,8576-8582$.

(19) Saini, M.; Sadhu, K. K. Two Instantaneous Fluorogenic Steps for Detection of Nanomolar Amyloid $\beta$ Monomer and Its Interaction with Stoichiometric Copper(II) Ion. Sens. Actuat. B Chem. 2020, 303, 127086.

(20) Fanni, A. M.; Monge, F. A.; Lin, C. Y.; Thapa, A.; Bhaskar, K.; Whitten, D. G.; Chi, E. Y. High Selectivity and Sensitivity of Oligomeric $p$-Phenylene Ethynylenes for Detecting Fibrillar and Prefibrillar Amyloid Protein Aggregates. ACS Chem. Neurosci. 2019, 10, 1813-1825.

(21) Tan, H. Y.; Zhou, K. X.; Yan, J. W.; Sun, H.; Pistolozzi, M.; Cui, M. C.; Zhang, L. Dual-Functional Red-Emitting Fluorescent Probes for Imaging $\beta$-Amyloid Plaques and Viscosity. Sens. Actuators B Chem. 2019, 298, 126903.

(22) Yan, J. W.; Zhu, J. Y.; Zhou, K. X.; Wang, J. S.; Tan, H. Y.; Xu, Z. Y.; Chen, S. B.; Lu, Y. T.; Cui, M. C.; Zhang, L. Neutral Merocyanine Dyes: For In Vivo NIR Fluorescence Imaging of Amyloid- $\beta$ Plaques. Chem. Commun. 2017, 53, 9910-9913.

(23) Watanabe, H.; Ono, M.; Saji, H. (2015). In Vivo Fluorescence Imaging of $\beta$-Amyloid Plaques with Push-Pull Dimethylaminothiophene Derivatives. Chem. Commun. 2015, 51, 17124-17127.

(24) Fu, H.; Peng, C.; Liang, Z.; Dai, J.; Liu, B.; Cui, M. In Vivo Near-infrared and Cerenkov Luminescence Imaging of Amyloid- $\beta$ Deposits in the Brain: A Fluorinated Small Molecule Used for Dual-modality Imaging. Chem. Commun. 2016, 52, 12745-12748. 
(25) Leung, C. W.; Guo, F.; Hong, Y.; Zhao, E.; Kwok, R. T.; Leung, N. L.; Chen, S.; Vaikath, N.

N.; El-Agnaf, O. M.; Tang, Y.; Gai, W. P.; Tang, B. Z. Detection of Oligomers and Fibrils of $\alpha$-Synuclein by AIEgen with Strong Fluorescence. Chem. Commun. 2015, 51, 1866-1869.

(26) Cheng, Y.; Zhu, B.; Deng, Y.; Zhang, Z. In Vivo Detection of Cerebral Amyloid Fibrils with Smart Dicynomethylene-4H-pyran-Based Fluorescence Probe. Anal. Chem. 2015, 87, 4781-4787.

(27) Staderini, M.; Martin, M. A.; Bolognesi, M. L.; Menendez, J. C. Imaging of $\beta$-Amyloid Plaques by Near Infrared Fluorescent Tracers: A New Frontier for Chemical Neuroscience. Chem. Soc. Rev. 2015, 44, 1807-1819.

(28) Hatai, J.; Motiei, L.; Margulies, D. Analyzing Amyloid $\beta$ Aggregates with a Combinatorial Fluorescent Molecular Sensor. J. Am. Chem. Soc. 2017, 139, 2136-2139.

(29) Yang, Y.; Li, S.; Zhang, Q.; Kuang, Y.; Qin, A.; Gao, M.; Li, F.; Tang, B. Z. An AIE-active Theranostic Probe for Light-up Detection of A $\beta$ Aggregates and Protection of Neuronal Cells. $J$. Mater. Chem. B 2019, 7, 2434-2441.

(30) Shin, J.; Verwilst, P.; Choi, H.; Kang, S.; Han, J.; Kim, N. H.; Choi, J. G.; Oh, M. S.; Hwang, J. S.; Kim, D.; Mook-Jung, I.; Kim, J. S. Harnessing Intramolecular Rotation To Enhance Two-Photon Imaging of A $\beta$ Plaques through Minimizing Background Fluorescence. Angew. Chem. Int. Ed. 2019, 58, 5648-5652.

(31) Hong, Y.; Meng, L.; Chen, S.; Leung, C. W.; Da, L. T.; Faisal, M.; Silva, D. A.; Liu, J.; Lam, J. W.; Huang, X.; Tang, B. Z. Monitoring and Inhibition of Insulin Fibrillation by a Small Organic Fluorogen with Aggregation-Induced Emission Characteristics. J. Am. Chem. Soc. 2012, 134, $1680-1689$.

(32) Jun, Y. W.; Cho, S. W.; Jung, J.; Huh, Y.; Kim, Y.; Kim, D.; Ahn, K. H. Frontiers in Probing Alzheimer's Disease Biomarkers with Fluorescent Small Molecules. ACS Cent. Sci. 2019, 5, 209-217.

(33) Benzeid, H.; Mothes, E.; Essassi, E.; Faller, P.; Pratviel, G. A Thienoquinoxaline and a Styryl-quinoxaline as New Fluorescent Probes for Amyloid- $\beta$ Fibrils. C. R. Chim. 2012, 15, $79-84$.

(34) Ono, M.; Watanabe, H.; Kimura, H.; Saji, H. BODIPY-based Molecular Probe for Imaging of Cerebral $\beta$-Amyloid Plaques. ACS Chem. Neurosci. 2012, 3, 319-324.

(35) Klunka, W. E.; Wang, Y. M.; Huang, G. -F.; Debnatha, M. L.; Holtb, D. P.; Mathis, C. A. 
Uncharged Thioflavin-T Derivatives Bind to Amyloid- $\beta$ Protein with High Affinity and Readily Enter the Brain. Life Sci. 2001, 69, 1471-1484.

(36) Luo, J.; Xie, Z.; Lam, J. W. Y.; Cheng,L.; Tang, B. Z.; Chen, H.; Qiu, C.; Kwok, H. S.; Zhan, X.; Liu, Y.; Zhu, D; Tang $\quad$ B. $\quad$ Z. Aggregation-Induced Emission of 1-Methyl-1,2,3,4,5-pentaphenylsilole. Chem. Commun. 2001, 1740-1741.

(37) Mei, J.; Hong, Y.; Lam, J. W.; Qin, A.; Tang, Y.; Tang, B. Z. Aggregation-Induced Emission: The Whole Is More Brilliant than the Parts. Adv. Mater. 2014, 26, 5429-5479.

(38) Pradhan, N.; Jana, D.; Ghorai, B. K.; Jana, N. R. Detection and Monitoring of Amyloid Fibrillation Using a Fluorescence "Switch-On" Probe. ACS Appl. Mater. Interfaces 2015, 7, $25813-25820$.

(39) Mei, J.; Huang, Y.; Tian, H. Progress and Trends in AIE-Based Bioprobes: A Brief Overview. ACS Appl. Mater. Interfaces 2018, 10, 12217-12261.

(40) Mei, J.; Leung, N. L.; Kwok, R. T.; Lam, J. W.; Tang, B. Z. Aggregation-Induced Emission: Together We Shine, United We Soar! Chem. Rev. 2015, 115, 11718-11940.

(41) Rajasekhar, K.; Narayanaswamy, N.; Murugan, N. A.; Kuang, G.; Agren, H.; Govindaraju, T. A High Affinity Red Fluorescence and Colorimetric Probe for Amyloid $\beta$ Aggregates. Sci. Rep. 2016, 6, 23668.

(42) Diner, I.; Dooyema, J.; Gearing, M.; Walker, L. C.; Seyfried, N. T. Generation of Clickable Pittsburgh Compound B for the Detection and Capture of $\beta$-Amyloid in Alzheimer's Disease Brain. Bioconj. Chem. 2017, 28, 2627.

(43) Zhu, S.; Wang, J.; Zhang, Y.; He, J.; Kong, J.; Wang, J. F.; Li, X. M. The Role of Neuroinflammation and Amyloid in Cognitive Impairment in an APP/PS1 Transgenic Mouse Model of Alzheimer's Disease. CNS Neurosci. Ther. 2017, 23, 310-320.

(44) Oakley, H.; Cole, S. L.; Logan, S.; Maus, E.; Shao, P.; Craft, J.; Guillozet-Bongaarts, A.; Ohno, M.; Disterhoft, J.; Van Eldik, L.; Berry, R.; Vassar, R. Intraneuronal $\beta$-Amyloid Aggregates, Neurodegeneration, and Neuron Loss in Transgenic Mice with Five Familial Alzheimer's Disease Mutations: Potential Factors in Amyloid Plaque Formation. J. Neurosci. 2006, 26, 10129-10140.

(45) Ametamey, S. M.; Honer, M.; Schubiger, P. A. Molecular Imaging with PET. Chem. Rev. 2008, 108, 1501. 
(46) Law, A. S.; Lee, L. C.; Yeung, M. C.; Lo, K. K.; Yam, V. W. Amyloid Protein-Induced Supramolecular Self-Assembly of Water-Soluble Platinum(II) Complexes: A Luminescence Assay for Amyloid Fibrillation Detection and Inhibitor Screening. J. Am. Chem. Soc. 2019, 141, $18570-18577$.

(47) Fan, D. Y.; Wang, Y. J. Early Intervention in Alzheimer's Disease: How Early is Early Enough? Neurosci. Bull. 2020, 36, 195-197.

(48) Liu, B.; Shen, H.; Hao, Y.; Zhu, X.; Li, S.; Huang, Y.; Qu, P.; Xu, M. Lanthanide Functionalized Metal-Organic Coordination Polymer: Toward Novel Turn-On Fluorescent Sensing of Amyloid $\beta$-Peptide. Anal. Chem. 2018, 90, 12449-12455.

(49) Lei, L.; Geng, R.; Xu, Z.; Dang, Y.; Hu, X.; Li, L.; Geng, P.; Tian, Y.; Zhang, W. Glycopeptide Nanofiber Platform for A $\beta$-Sialic Acid Interaction Analysis and Highly Sensitive Detection of A $\beta$. Anal. Chem. 2019, 91, 8129-8136.

(50) Kumar, M.; Hong, Y.; Thorn, D. C.; Ecroyd, H.; Carver, J. A. Monitoring Early-Stage Protein Aggregation by an Aggregation-Induced Emission Fluorogen. Anal. Chem. 2017, 89, 9322-9329. (51) Yan, X.; Wei, P.; Liu, Y.; Wang, M.; Chen, C.; Zhao, J.; Li, G.; Saha, M. L.; Zhou, Z.; An, Z.; Li, X.; Stang, P. J. Endo- and Exo-Functionalized Tetraphenylethylene $\mathrm{M}_{12} \mathrm{~L}_{24}$ Nanospheres: Fluorescence Emission inside a Confined Space. J. Am. Chem. Soc. 2019, 141, 9673-9679.

(52) Liu, H.; Qian, C. Y.; Yang, T.; Wang, Y. Q.; Luo, J.; Zhang, C. L.; Wang, X. H.; Wang, X. Y.; Guo, Z. J. Small Molecule-Mediated Co-Assembly of Amyloid $\beta$ Oligomers Reduces Neurotoxicity Through Promoting Non-Fibrillar Aggregation. Chem. Sci. 2020, 11, 7158-7169.

(53) Liu, Y.; Yang, Y.; Sun, M.; Cui, M.; Fu, Y.; Lin, Y.; Li, Z.; Nie, L. Highly Specific Noninvasive Photoacoustic and Positron Emission Tomography of Brain Plaque with Functionalized Croconium Dye Labeled by a Radiotracer. Chem. Sci. 2017, 8, 2710-2716.

(54) Veloso, A. J.; Chow, A. M.; Ganesh, H. V.; Li, N.; Dhar, D.; Wu, D. C.; Mikhaylichenko, S.; Brown, I. R.; Kerman, K. Electrochemical Immunosensors for Effective Evaluation of Amyloid- $\beta$ Modulators on Oligomeric and Fibrillar Aggregation Process. Anal. Chem. 2014, 86, 4901-4909.

(55) Fu, H.; Cui, M.; Zhao, L.; Tu, P.; Zhou, K.; Dai, J.; Liu, B. Highly Sensitive Near-Infrared Fluorophores for In Vivo Detection of Amyloid- $\beta$ Plaques in Alzheimer's Disease. J. Med. Chem. 2015, 58, 6972-6983.

(56) Zhao, D. S.; Chen, Y. X.; Liu, Q.; Zhao, Y. F.; Li, Y. M. Exploring the Binding Mechanism of 
Thioflavin-T to the $\beta$-Amyloid Peptide by Blind Docking Method. Sci. China Chem. 2011, 55, $112-117$.

\section{Table of Contents Graphic}

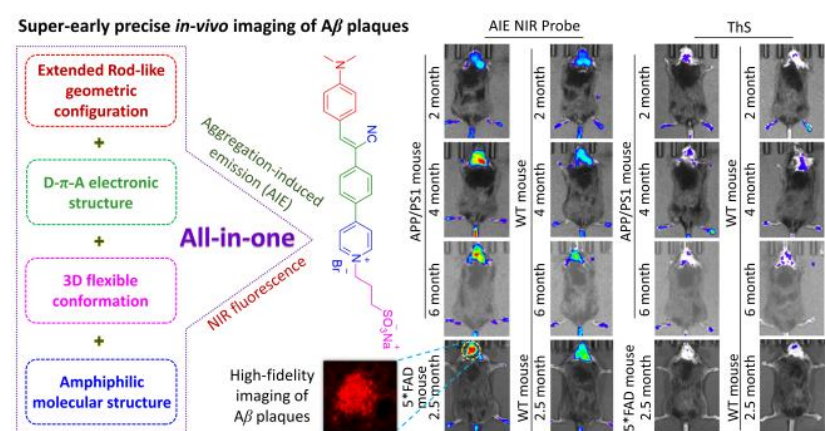

